\title{
Genital chlamydial infection: association between clinical features, organism genotype and load
}

\author{
Correspondence \\ Hamid Jalal \\ hamid.jalal@addenbrookes.nhs.uk
}

Received 11 November 2010

Accepted 15 March 2011

\author{
Hamid Jalal, ${ }^{1}$ Neville O. Verlander, ${ }^{2}$ Navin Kumar, ${ }^{1}$ Neil Bentley, ${ }^{1}$ \\ Christopher Carne $^{3}$ and Christopher Sonnex ${ }^{3}$
}

\author{
${ }^{1}$ Clinical Microbiology and Public Health Laboratory, Health Protection Agency, Addenbrooke's \\ Hospital, Hills Road, Cambridge CB2 00W, UK \\ ${ }^{2}$ Statistics Unit, Statistics, Modelling and Economics Department, Centre for Infections, Health \\ Protection Agency, London, UK \\ ${ }^{3}$ Department of Genitourinary Medicine, Addenbrooke's Hospital, Hills Road, Cambridge CB2 20O, \\ UK
}

The association between the clinical features of genital chlamydial infection and organism genotype and load was evaluated. Chlamydial DNA was detected and quantified in genital swabs from $233(7 \%)$ of 3384 consecutive patients attending a genitourinary medicine clinic. The chlamydia-positive subcohort comprised 132 (57\%) females and 101 (43\%) males. Clinical features were present in $33 \%$ women and $72 \%$ men. The chlamydial load was found to be higher in women (median load: $5.6 \mathrm{log}$ ) than men (median load: $3.5 \mathrm{log}$ ). Single variable analysis failed to show a significant association between chlamydial load and clinical features $(P$ value $=0.3)$. Owing to the limited amount of clinical material, information on chlamydial genotypes was available for $70 \%(n=162)$ of chlamydia-positive patients. However, multivariable analysis of these samples did show a significant association between chlamydial load and clinical features $(P$ value $=0.02)$. This discrepancy is most probably due to the difference in the amount of data analysed by single variable (data from 233 patients) and multivariable (data from 162 patients) analysis. The distribution of chlamydia genotypes was as follows: type $E(46 \%), F(22 \%)$, D (8\%), K (8\%), G (7\%), J (4\%), I (1\%) and H (0.6\%). No statistically significant association was observed between chlamydial genotype and clinical features in either single variable $(P$ value $=0.6)$ or multivariable $(P$ value $=0.4)$ analysis. These findings suggest that chlamydial load and diversity in the ompA gene plays little, if any, role in the pathogenesis of genital chlamydial infection.

\section{INTRODUCTION}

The role of organism load in determining the clinical presentation of genital Chlamydia trachomatis (CT) infection has been a focus of interest over the past several years. Studies using CT culture and utilizing the technique of quantifying the number of c.f.u. have found higher loads in younger patients, in women compared to men and in those with evidence of genital tract inflammation (Eckert et al., 2000; Geisler et al., 2001). In contrast, molecular studies have reported variable results. Chlamydial load has been positively associated with symptoms and clinical signs in men but only the presence of multiple symptoms or clinical signs has been associated with organism load in

Abbreviations: $\mathrm{BHQ}$, black-hole quencher; $\mathrm{Cl}$, confidence interval; $\mathrm{CMO}$ EAG, Chief Medical Officer's Expert Advisory Group; CT, Chlamydia trachomatis; FAM, 6-carboxyfluorescein; GU, genitourinary; MGB, minor groove binder; NFQ, non-fluorescent quencher; OR, odds ratio; ROX, carboxy-X-rhodamine. women (Michel et al., 2007). Two studies have reported no association between clinical features and chlamydial load in first void urine specimens from men or women (Gomes et al., 2006; Wiggins et al., 2009). Chlamydial load in vulvovaginal swabs was reported to be higher among women with vaginal discharge as compared to women without discharge (Wiggins et al., 2009).

Considering the role of chlamydial genotypes or serovars in determining clinical presentation, serovar $\mathrm{Ga}$ has been found to be associated with dysuria in men, serovar $\mathrm{F}$ with abdominal pain and/or dyspareunia in women and serovar $\mathrm{K}$ with vaginal discharge (Geisler et al., 2003; Morré et al., 2000). Serovar Ia has been detected more frequently in asymptomatic patients. There appears to be no association between serovars $\mathrm{D}$ and $\mathrm{E}$ and either symptomatic or asymptomatic infection. CT serovars D, E, F, I and J were the most frequently detected types in these two studies. Young white women infected with B class serovars (B, D, 
$\left.\mathrm{D}^{-}, \mathrm{E}\right)$ have also been found to yield the highest organism load (Eckert et al., 2000). Other studies using either serological or molecular techniques to type CT have reported either no (Batteiger et al., 1989; Gomes et al., 2006; Millman et al., 2006; Van de Laar et al., 1996) or weak (Boisvert et al., 1999; Dean et al., 1995; Van Duynhoven et al., 1998; Workowski et al., 1994) association between CT type and the presence or absence of clinical features.

This study was designed to overcome the two limitations of previous molecular studies (Gomes et al., 2006; Michel et al., 2007; Wiggins et al., 2009), i.e. non-consecutive patients and the adverse effect of sample storage on chlamydial load. We investigated the association between clinical features and chlamydial load with and without genotype identification by using real-time PCR on fresh genital swabs from consecutive patients attending a genitourinary (GU) medicine clinic.

\section{METHODS}

Study population. The study population comprised 3384 consecutive patients attending the Department of Genitourinary Medicine at Addenbrooke's Hospital, Cambridge, UK, from August 2005 to January 2006. There were 1640 males [median age: 26 years (range: 13-73)] and 1744 females [median age: 23 years (range: $12-66$ )] in this cohort. Two swabs (endocervical and urethral) were obtained from female patients, placed in a single tube and treated as a single sample. The urethral sample was taken from just within the urethra. The cervix was cleaned to remove excess mucus prior to taking the cervical sample. The sample was collected by inserting a cotton swab into the cervical os, rotating firmly, withdrawing and then placing the swab in chlamydia transport medium (CTM). A single urethral swab was analysed from each male patient. This was obtained by inserting a cotton swab 2-4 cm into the urethra, rotating firmly, withdrawing and placing the swab into CTM. All swabs for chlamydia testing were collected after taking swabs for microscopy and Neisseria gonorrhoeae culture. All samples were collected by experienced practitioners trained in sample collection. The IDEIA chlamydia specimen collection kit (S600730; Dako) was used for the collection of genital swabs as described previously (Jalal et al., 2006a). Urine specimens were not used for chlamydia detection. Clinical data were recorded for each patient and linked to chlamydia quantification and genotyping results by a code. To ensure anonymity, patient identifiers were removed after coding. Men were classified as symptomatic if they presented with dysuria, urethral discharge or intra-scrotal pain and women if they had symptoms of vaginal discharge, dysuria, pelvic discomfort/pain, or post-coital or intermenstrual bleeding. A record was also made of the presence of cervicitis, defined as mucopurulent secretions with or without contact bleeding. Quantification of polymorphs on microscopy of a Gram-stained smear of cervical secretions was not used to diagnose cervicitis. The presence of urethritis in men, defined as $\geqslant 5$ polymorphs on microscopy $(\times 1000$ magnification) of a Gram-stained urethral smear, was also recorded. The Cambridge Local Research Ethics Committee approved this study.

Chlamydial load and genotyping. The Clinical Microbiology and Public Health Laboratory uses an in-house quantitative real-time PCR for the detection and quantification of CT DNA in clinical samples. This is a triplex assay targeting cryptic plasmid (qualitative detection), ompA gene (quantitative detection and confirmation) and an internal control (synthetic DNA molecule to quality control DNA extraction and amplification) (Jalal et al., 2006b). Three changes were made to the published method: (i) DNA from swabs was extracted using CAS1820 X-Tractor Gene (Corbett Robotics) instead of MagNa Pure LC Robot (Roche Diagnostics); (ii) the number of amplification cycles was increased from 40 to 45 ; and (iii) $\mathrm{HJ}$ plasmid probe was modified (5'-FAM-CGAACTCATCGGCG-MGB-NFQ-3'; this probe was synthesized by Applied Biosystems). The performance of the assay after these changes was validated on three consecutive runs, each containing 94 samples and controls as described previously (Jalal et al., 2006b). These modifications did not affect the performance of the assay. Agreed turn-around time for CT quantitative results was $\leqslant 5$ days. After the completion of routine diagnostic work, 162 (70\%) of 233 samples contained sufficient material to determine CT genotype. These leftover samples were stored at $-20{ }^{\circ} \mathrm{C}$ and CT genotyping was performed by using nested real-time PCR as described previously (Jalal et al., 2007a).

rpoB PCR. The routine diagnostic assay targets the ompA gene for the quantification of chlamydia genomic DNA. The primers and probe for this part of the triplex assay are from highly conserved regions of the ompA gene. However, there are a few nucleotide variations in the amplicons from different genotypes. We wanted to investigate the effect of these variations on the quantitative ability of the current assay. Hence, a new assay was designed targeting the rpoB gene with no nucleotide variation in amplicons from different genotypes of chlamydia. $r p o B$ PCR was used to investigate the effect of sample storage at $-20{ }^{\circ} \mathrm{C}$. After genotyping, 148 samples contained sufficient material for this analysis. This uniplex assay targeted the $r p o \mathrm{~B}$ gene of $\mathrm{CT}$. The new assay was optimized to produce comparable sensitivity with the quantitative part (ompA) of the routine diagnostic assay. The concentration of all PCR ingredients and the amplification parameters of the new assay were the same as those for the routine diagnostic assay except that different primers and probe were used (Jalal et al., 2006b): forward primer, 5' ACGACCAGGAGAGCCTGCAA-3' (location: 1421); reverse primer, 5'-ATAACGTCCAACGCGGCCTA-3' (location: 1511); and probe, 5'-ROX-TGCTCGATCCACAATTATGCG-BHQ-2-3' (location: 1442). The locations of primers and probe were according to GenBank accession no. AY826975. These primers and the probe were synthesized by metabion International. The lower limit of detection for $r p o B$ PCR was determined by testing a dilution series of CT type E ATCC VR-348B at the following concentrations: $12,6,3,1.5$ and 0.75 genome copies per reaction. Each concentration was tested 10 times and Probit analysis of the results was carried out using StatsDirect version 2.4.1. A total of 14 isolates representing all the genovars of CT and 26 bacterial isolates representing the pathogenic bacteria and commensals of the oropharynx, genital tract and rectum were used to investigate the specificity of the primers and probe. The identity of these organisms is shown in a previous publication (Jalal et al., 2006b - in Tables 1 and 2).

Statistical analysis. The chlamydial load values were converted to natural logarithms and logged values were used in statistical analysis of the data. Two main sets of analyses were performed: one where chlamydial load was the outcome (continuous) and the other where the presence or absence of clinical features was the outcome (binary). The Kruskal-Wallis test was employed to investigate the association between age and CT genotypes. Censored normal linear regression was used when the outcome was continuous, and logistic regression was used for the binary outcome; $P$ values were determined by the likelihood ratio test. Each explanatory variable was examined consecutively for an association with the outcome in single variable analysis. This was followed by multivariable analysis in a backwards stepwise procedure wherein the least significant variable was removed at each step, provided it was not a significant confounder, i.e. its removal resulted in a change [in the coefficient for the continuous outcome and odds ratio (OR) when the outcome was binary] of less 
than $10 \%$. This continued until all variables were either significant at the $5 \%$ level or were substantial confounders, at which point interactions between the significant variables were added to the model and removed one at a time if not significant. When using clinical features as the outcome, the logged chlamydial loads were grouped into the following bands: less than 3, 3 to less than 4,4 to less than 5, 5 to less than 6 and 6 or more. All analyses were performed in STATA 11 .

\section{RESULTS}

\section{Effect of sample storage at $-20^{\circ} \mathrm{C}$ on chlamydial load}

According to Probit analysis, rpoB PCR had a $95 \%$ probability of detection at 2 [confidence interval (CI) of 1 to 4] genome copies of CT per reaction. The primers and probe used in $r p o B$ PCR did not cross-react with DNA from any of the pathogenic bacteria or commensals from the oropharynx, genital tract and rectum that were tested. $r p o B$ PCR amplified DNA from all strains of CT. Although CT DNA was detected in all of the 148 samples before and after storage, there was a significant degradation of DNA in almost all samples after storage at $-20{ }^{\circ} \mathrm{C}$. There were only three samples with a load of $<1 \log _{10}$ genome copies (ml $\mathrm{CTM})^{-1}$ before storage. This number increased to 27 after storage. Similarly, there were 40 samples with a load of $>6 \log$ before storage. Only one sample had a load of $>6 \log$ after storage. A comparison of chlamydial load before and after storage is shown in Fig. 1. We were unable to use $r p o B$ PCR for its intended purpose due to the limited quantity of DNA in stored samples. The chlamydial load data from stored samples were not used to investigate the association between clinical features and chlamydial load.

\section{Association between covariates (sex, age and genotype) and chlamydial load}

CT DNA was detected in genital swabs from 233 (7\%) of 3384 patients. This CT-positive subcohort comprised 132 (57\%) women [median age: 20 years (range: $15-44)$ ] and $101(43 \%)$ men [median age: 23 years (range: 17-62)]. The accurate quantification of chlamydial load was not possible for six women and three men due to the low quantity of CT DNA in the samples from these patients. Samples from 54 women and 2 men had chlamydial loads of $>6 \log$. Due to limited resources and a fixed turn-around time of $\leqslant 5$ days, these samples were not further titrated to determine the precise value of the chlamydial load.

Genital swabs from women (median load: $5.6 \mathrm{log}$ ) had a much higher chlamydial load than those from men (median load: $3.5 \mathrm{log}$ ). The distribution of chlamydial load among women and men is shown in Fig. 2. To investigate the effect of age on chlamydial load, the age of participants was categorized into the following bands in line with a published study (Gomes et al., 2006): $\leqslant 20$ years, $21-30,31-40$ and $>41$ years. The majority of CT-positive patients were under the age of 30 years [95.4\%

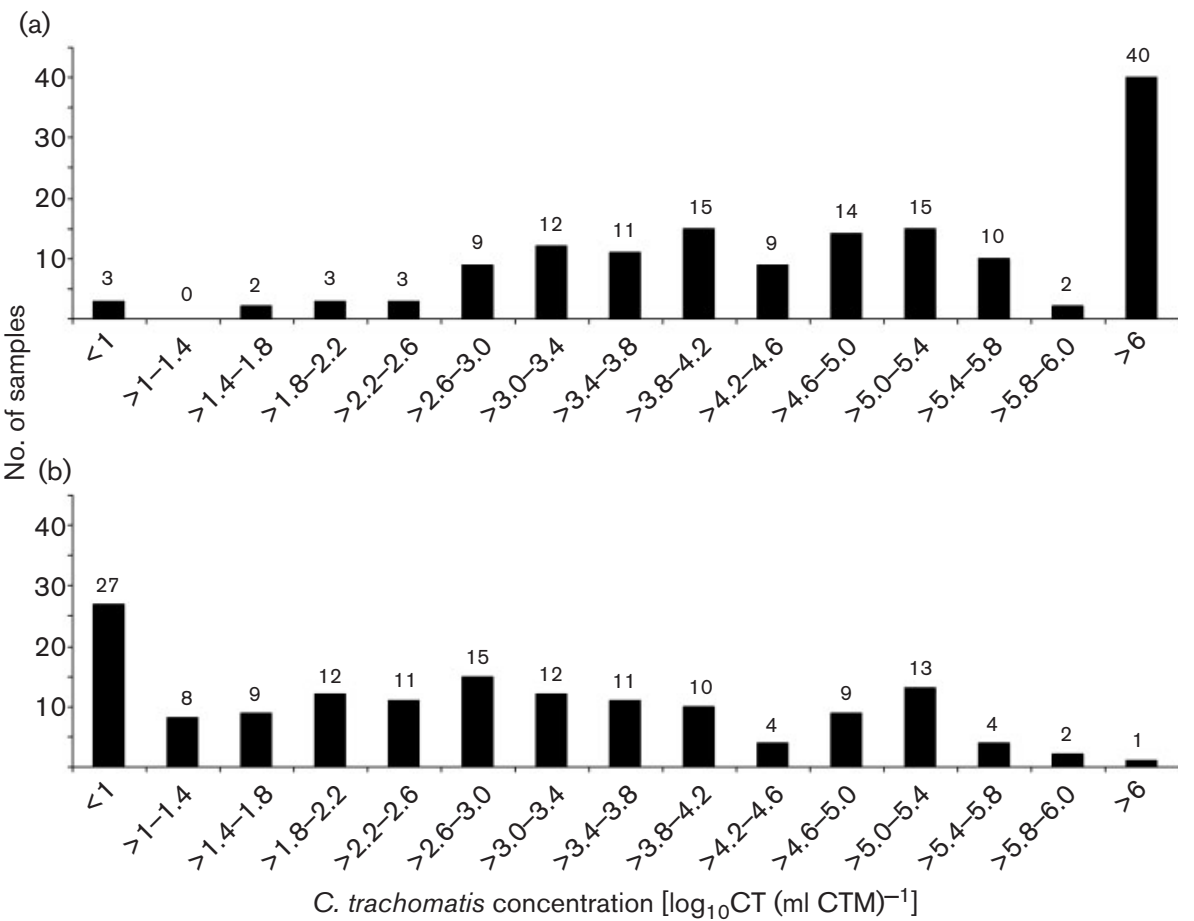

Fig. 1. Histograms showing comparative load for 148 samples before (a; ompA PCR) and after (b; rpoB PCR) storage at $-20{ }^{\circ} \mathrm{C}$. 


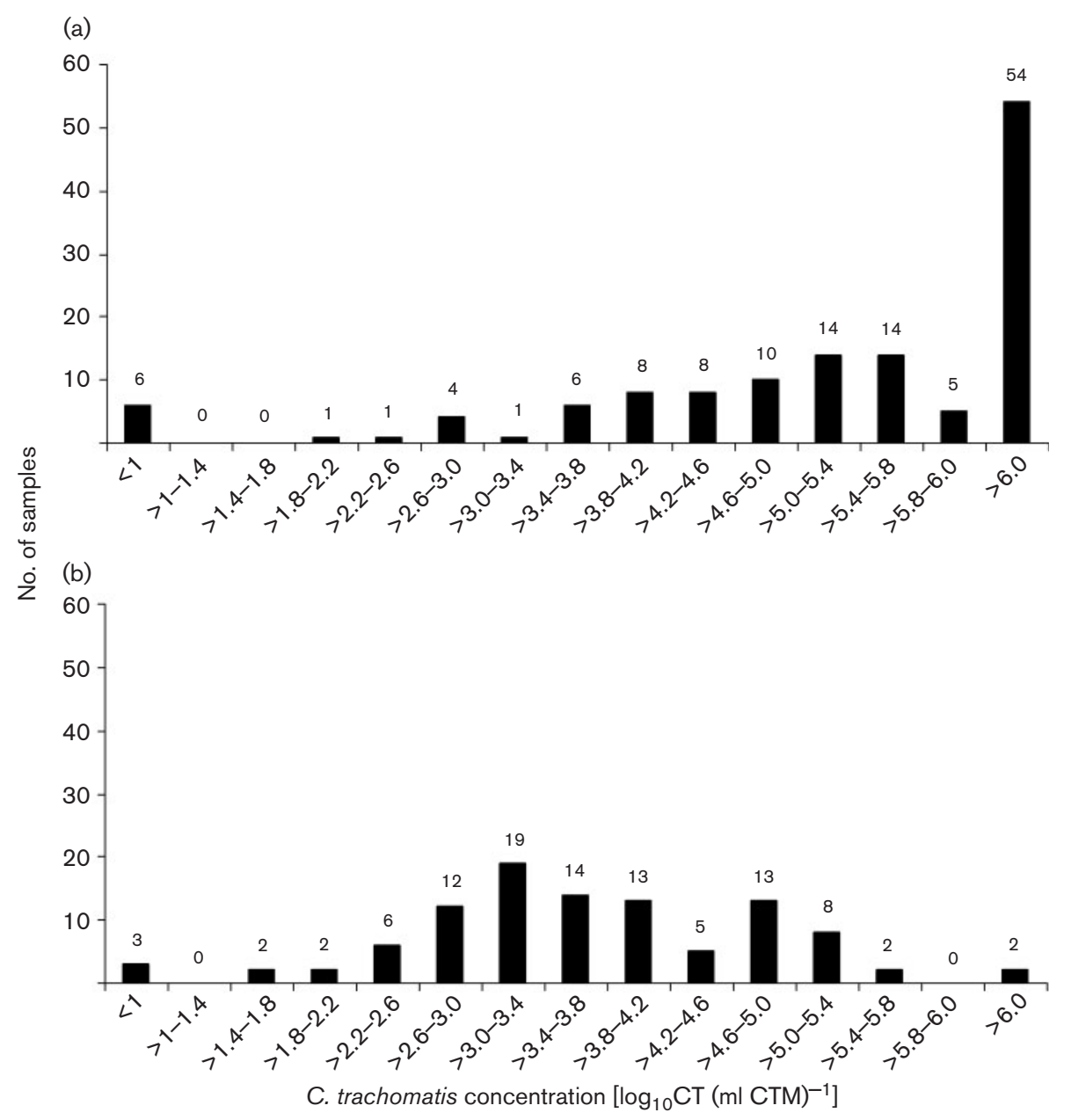

Fig. 2. Histograms of the distribution of clinical samples based on the quantification of CT ompA copy number ( $\mathrm{ml} C T M)^{-1}$. Chlamydial loads in (a) 132 women and (b) 101 men are shown.

( $n=126)$ women and $78 \%(n=79)$ men]. While there was a weak association between age groups and chlamydial load in single variable analysis $(P=0.09)$, with the $21-30$ year age group having lower load than those aged under 20 , this association was not apparent on multivariable analysis $(P=0.5)$ (Table 1).

CT genotyping results were available for $162(70 \%)$ of the 233 chlamydia-positive patients. This group comprised 90 women and 72 men. The distribution of CT genotypes was as follows: type E (46\%), F (22\%), D (8\%), K (8\%), G (7\%), J (4\%), I ( $1 \%)$ and $\mathrm{H}(0.6 \%)$. Two different genotypes were detected in swabs from four patients $(2 \%)$. There was no significant difference in the distribution of CT genotype between women and men. The KruskalWallis test revealed no association between age and genotype $(P=0.4)$. Table 2 shows the distribution of genotypes between different age groups (a Fisher's exact test yielded a $P$ value of 0.2 ). Similarly, there was no association between genotype and chlamydial load ( $P$ value of 0.6). The results of single and multivariable censored regression analysis are summarized in Table 1. These results demonstrated that only gender was associated with chlamydial load, with males having a significantly lower load than females.

\section{Association between covariates (sex, age, chlamydial load and genotype) and clinical features}

Clinical features of genital infection were found in 44 $(33 \%)$ of 132 women and $73(72 \%)$ of 101 men. Signs of cervicitis and urethritis as defined in Methods were identified in $10(23 \%)$ of the 44 women and $49(67 \%)$ of the 73 men, respectively. All women with cervicitis had a chlamydial load of $>4.3 \mathrm{log}$ and seven of them had a load of $>6 \log$. A total of 42 of the 49 men with urethritis had a chlamydial load of $\geqslant 3 \mathrm{log}$. There was a strong association between sex and clinical features, with the OR $(<0.001)$ for males to have clinical features being much greater than for 
Table 1. The association of covariates (sex, age and genotype) with chlamydial load

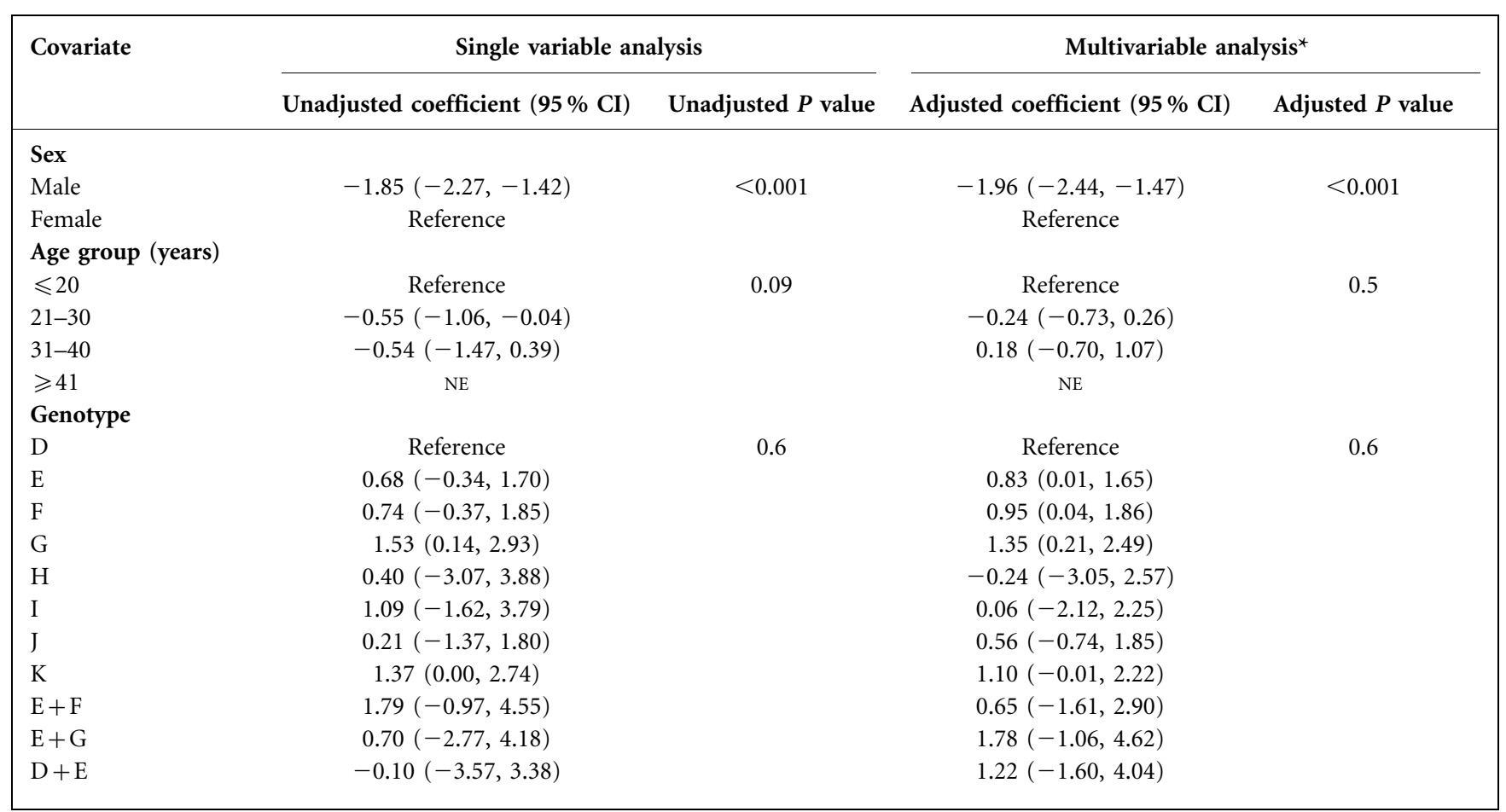

NE, Not estimable.

${ }^{\star}$ Adjusted results based on 162 observations.

females. No significant association was found between age and CT genotype and clinical features. Single variable analysis suggested no association between CT load and clinical features, whereas multivariable analysis indicated otherwise. This difference may be due to the smaller number of observations available in the multivariable analysis. The results of single and multivariable analysis for the association between covariates (sex, age, load and genotype) and clinical features are summarized in Table 3.

Table 2. The distribution of age groups and genotypes

\begin{tabular}{|lccc|}
\hline Genotype & \multicolumn{3}{c|}{ Age group (years) } \\
\cline { 2 - 4 } & $\leqslant \mathbf{2 0}$ & $\mathbf{2 1 - 3 0}$ & $\mathbf{3 1 - 4 0}$ \\
\hline $\mathrm{D}$ & 5 & 7 & 1 \\
$\mathrm{E}$ & 24 & 42 & 9 \\
$\mathrm{~F}$ & 20 & 14 & 1 \\
$\mathrm{G}$ & 5 & 5 & 2 \\
$\mathrm{H}$ & 0 & 1 & 0 \\
$\mathrm{I}$ & 2 & 0 & 0 \\
$\mathrm{~J}$ & 4 & 3 & 0 \\
K & 8 & 5 & 0 \\
E $+\mathrm{F}$ & 1 & 0 & 1 \\
E + G & 1 & 0 & 0 \\
D + E & 0 & 1 & 0 \\
Total & 70 & 78 & 14 \\
\hline
\end{tabular}

\section{DISCUSSION}

This study investigated the association between chlamydial load in genital swabs with ompA genotypes and clinical features. The design of this study is similar to that performed by Gomes et al. (2006) with a few exceptions. Apart from different study populations, Gomes et al. (2006) performed their analysis on stored DNA from first void urine. This approach was based on the evidence for chlamydial coinfection of the urethra and cervix in women. However, the chlamydial load in urine may not be a true reflection of the cervical chlamydial load. Their study reported a wide distribution in the number of epithelial cells ( $\mathrm{ml}$ urine $)^{-1}$ suggesting a variation in the time between urine collection and last previous urination. Using a variable denominator (epithelial cells) to normalize the chlamydial load may affect the accuracy of quantitative data. For this reason, genital swabs were used for quantitative analysis in this study. We did not normalize the chlamydial load data by the quantification of cellular DNA because genital swabs contain a mixture of different types of cells, i.e. columnar, squamous and inflammatory cells. Normalizing chlamydial load by total genomic DNA load does not provide the relevant information, i.e. chlamydial load per columnar cell. The potential bias in our quantitative data, due to differences in sample collection by nurses and doctors, was minimized by a standardized method of sample collection.

Quantification of chlamydial DNA was performed on fresh samples as part of the routine diagnostic service. The 
Table 3. The association of covariates (sex, age, chlamydial load and genotype) with clinical features

\begin{tabular}{|c|c|c|c|c|c|c|}
\hline \multirow[t]{2}{*}{ Covariate } & \multicolumn{2}{|c|}{ Clinical features } & \multicolumn{2}{|c|}{ Single variable analysis } & \multicolumn{2}{|c|}{ Multivariable analysis ${ }^{\star}$} \\
\hline & Presence & Absence & $\begin{array}{l}\text { Unadjusted OR } \\
\quad(95 \% \mathrm{CI})\end{array}$ & $\begin{array}{l}\text { Unadjusted } P \\
\text { value }\end{array}$ & $\begin{array}{l}\text { Adjusted OR } \\
(95 \% \mathrm{CI})\end{array}$ & $\begin{array}{l}\text { Adjusted } P \\
\text { value }\end{array}$ \\
\hline \multicolumn{7}{|l|}{ Sex } \\
\hline Male & 73 & 28 & $5.21(2.96,9.19)$ & $<0.001$ & $13.87(4.46,43.20)$ & $<0.001$ \\
\hline Female & 44 & 88 & Reference & & Reference & \\
\hline \multicolumn{7}{|c|}{ Age group (years) } \\
\hline$\leqslant 20$ & 45 & 65 & Reference & 0.03 & Reference & 0.17 \\
\hline $21-30$ & 62 & 43 & $2.08(1.21,3.59)$ & & $1.59(0.73,3.45)$ & \\
\hline $31-40$ & 10 & 8 & $1.81(0.66,4.93)$ & & $0.48(0.11,2.09)$ & \\
\hline$\geqslant 41$ & 0 & 0 & $\mathrm{NE}$ & & $\mathrm{NE}$ & \\
\hline \multicolumn{7}{|c|}{$\begin{array}{l}\text { Chlamydial load } \\
\text { (logged) }\end{array}$} \\
\hline$<3$ & 17 & 20 & Reference & 0.3 & Reference & 0.02 \\
\hline 3 to $<4$ & 28 & 24 & $1.37(0.59,3.20)$ & & $1.05(0.30,3.64)$ & \\
\hline 4 to $<5$ & 28 & 17 & $1.94(0.80,4.69)$ & & $3.08(0.76,12.50)$ & \\
\hline 5 to $<6$ & 20 & 23 & $1.02(0.42,2.47)$ & & $4.44(1.02,19.41)$ & \\
\hline$\geqslant 6$ & 24 & 32 & $0.88(0.38,2.03)$ & & $7.92(1.73,36.28)$ & \\
\hline \multicolumn{7}{|l|}{ Genotype } \\
\hline $\mathrm{D}$ & 6 & 7 & Reference & 0.6 & Reference & 0.4 \\
\hline $\mathrm{E}$ & 43 & 32 & $1.57(0.48,5.12)$ & & $1.24(0.31,4.92)$ & \\
\hline $\mathrm{F}$ & 16 & 19 & $0.98(0.27,3.52)$ & & $0.64(0.14,2.91)$ & \\
\hline G & 6 & 6 & $1.17(0.24,5.62)$ & & $1.09(0.18,6.58)$ & \\
\hline $\mathrm{H}$ & 1 & 0 & $\mathrm{NE}$ & & $\mathrm{NE}$ & \\
\hline I & 1 & 1 & $1.17(0.06,22.94)$ & & $3.43(0.11,111)$ & \\
\hline $\mathrm{J}$ & 3 & 4 & $0.87(0.14,5.58)$ & & $0.72(0.09,6.06)$ & \\
\hline K & 6 & 7 & $1.00(0.21,4.67)$ & & $0.88(0.14,5.71)$ & \\
\hline$E+F$ & 2 & 0 & $\mathrm{NE}$ & & $\mathrm{NE}$ & \\
\hline $\mathrm{E}+\mathrm{G}$ & 1 & 0 & $\mathrm{NE}$ & & $\mathrm{NE}$ & \\
\hline $\mathrm{D}+\mathrm{E}$ & 1 & 0 & $\mathrm{NE}$ & & $\mathrm{NE}$ & \\
\hline
\end{tabular}

NE, Not estimable.

${ }^{\star}$ Adjusted results based on 162 observations.

accuracy of quantification data on stored samples or DNA is undetermined. Two studies investigating chlamydial load have reported considerable decline in the positivity rate of samples after the storage of extracted DNA (Gomes et al., 2006) or unextracted clinical material (Wiggins et al., 2009) at $-20{ }^{\circ} \mathrm{C}$. A total of $54(22 \%)$ of the 244 Amplicorpositive urine samples did not amplify using ompA nested PCR (Gomes et al., 2006). Similarly, RealArt PCR (Qiagen) did not detect CT DNA in $102(21.4 \%)$ of 476 Amplicorpositive samples (Wiggins et al., 2009). The authors concluded that it was not possible to rule out degradation of DNA, in spite of the difference in sensitivity between the qualitative (target: cryptic plasmid, higher sensitivity due to multiple copies) and the quantitative (target: ompA gene, lower sensitivity due to a single copy) PCRs. In this study, a significant degradation in CT DNA in genital swabs was observed after storage at $-20{ }^{\circ} \mathrm{C}$, even when quantitative PCRs of comparable sensitivity were performed on these samples (Fig. 1). A further strength of our study was the unbiased recruitment of patients and complete load dataset for the chlamydia-positive subcohort. The weaknesses of our study included a single centre for patient recruitment and an incomplete genotyping dataset due to limited clinical material. To avoid the recruitment bias, it was decided not to collect extra samples for the purpose of this study. Hence, the investigations for the association between chlamydial load with genotypes and clinical features were possible for $162(70 \%)$ patients, but the data for chlamydial load, clinical features and other covariates were available for all of the 233 chlamydia-positive patients. The distribution of genotypes in our cohort was similar to that given in a number of published studies from the USA and different European countries (Jalal et al., 2007b; Lysén et al., 2004; Oehme et al., 2003; Sayada et al., 1992; Suchland et al., 2003).

Gomes et al. (2006) reported that gender was not associated with a difference in chlamydial load. We report a higher median load ( 2 logs) for women than for men. Gomes et al. (2006) used urine from both men and women to represent loads at one anatomical site, i.e. the urethra. In our study, the load for women represented a cumulative load at two different anatomical sites, i.e. the cervix and 
urethra, and that for men represented the load at a single anatomical site, i.e. the urethra. A higher quantity of material in samples from women may have resulted in this finding. A culture-based study that used genital swabs for the quantification of chlamydial DNA reported a sixfold higher load for women (Eckert et al., 2000). Although the discomfort associated with urethral swab collection from men may contribute to the lower load, there are important biological differences between men and women that may have a significant effect on the load. These biological differences include the presence of a greater amount of infection-susceptible epithelium in women and the chlamydial-killing effects of prostatic secretions and the urethral flushing effect of urination in men (Eckert et al., 2000). Culture-based studies have reported a higher chlamydial load at a younger age (Eckert et al., 2000; Geisler et al., 2001). We did not observe any effect of age on load. The immunological principle behind the decrease in load among older patients is partial immunity due to repeated infections. Since 2005, genital chlamydial infection has been diagnosed by nucleic acid amplification techniques for 2239 (7\%) of 31689 patients attending the GU Medicine Clinic at Addenbrooke's Hospital. The median prevalence of genital chlamydial infection among patients attending GU clinics in the UK was reported to be $16.4 \%$ (range: 7-29) (CMO EAG, 1998). The prevalence of infection is relatively low in the population attending our GU clinic. This local epidemiology may suggest a lower prevalence of repeated infections among our patients rather than a lower load with increasing age. Gomes et al. (2006) reported no effect of age on chlamydial load and no association between clinical features and chlamydial load and genotype.

In summary, we failed to find an association between different chlamydial genotypes and the presence or absence of clinical features. Similarly, single variable analysis did not show a significant association between chlamydial load and clinical features. However, multivariable analysis showed a significant association between chlamydial load and clinical features. This discrepancy was most probably due to the difference in the amount of data analysed by single variable (233 datasets) and multivariable (162 datasets) methods. These results differ from those of culture-based studies (Eckert et al., 2000; Geisler et al., 2001), but are consistent with a published molecular study (Gomes et al., 2006). The lack of association between CT genotypes and clinical features suggests that the polymorphism in ompA does not play a significant role in pathogenicity. Similarly, the lack of association between chlamydial load and clinical features indicates the importance of host factors in the pathogenesis of infection. Based on these observations, we have designed future genomic and proteomic studies to investigate the pathogenesis of genital chlamydial infection.

\section{REFERENCES}

Batteiger, B. E., Lennington, W., Newhall, W. J., V, Katz, B. P., Morrison, H. T. \& Jones, R. B. (1989). Correlation of infecting serovar and local inflammation in genital chlamydial infections. $J$ Infect Dis 160, 332-336.

Boisvert, J. F., Koutsky, L. A., Suchland, R. J. \& Stamm, W. E. (1999). Clinical features of Chlamydia trachomatis rectal infection by serovar among homosexually active men. Sex Transm Dis 26, 392-398.

CMO EAG (1998). Main Report of the CMO's Expert Advisory Group on Chlamydia trachomatis. London: Department of Health.

Dean, D., Oudens, E., Bolan, G., Padian, N. \& Schachter, J. (1995). Major outer membrane protein variants of Chlamydia trachomatis are associated with severe upper genital tract infections and histopathology in San Francisco. J Infect Dis 172, 1013-1022.

Eckert, L. O., Suchland, R. J., Hawes, S. E. \& Stamm, W. E. (2000). Quantitative Chlamydia trachomatis cultures: correlation of chlamydial inclusion-forming units with serovar, age, sex, and race. J Infect Dis 182, 540-544.

Geisler, W. M., Suchland, R. J., Whittington, W. L. \& Stamm, W. E. (2001). Quantitative culture of Chlamydia trachomatis: relationship of inclusion-forming units produced in culture to clinical manifestations and acute inflammation in urogenital disease. J Infect Dis 184, 13501354.

Geisler, W. M., Suchland, R. J., Whittington, W. L. \& Stamm, W. E. (2003). The relationship of serovar to clinical manifestations of urogenital Chlamydia trachomatis infection. Sex Transm Dis 30, 160165.

Gomes, J. P., Borrego, M. J., Atik, B., Santo, I., Azevedo, J., Brito de Sá, A., Nogueira, P. \& Dean, D. (2006). Correlating Chlamydia trachomatis infectious load with urogenital ecological success and disease pathogenesis. Microbes Infect 8, 16-26.

Jalal, H., Stephen, H., Al-Suwaine, A., Sonnex, C. \& Carne, C. (2006a). The superiority of polymerase chain reaction over an amplified enzyme immunoassay for the detection of genital chlamydial infections. Sex Transm Infect 82, 37-40.

Jalal, H., Stephen, H., Curran, M. D., Burton, J., Bradley, M. \& Carne, C. (2006b). Development and validation of a rotor-gene real-time PCR assay for detection, identification, and quantification of Chlamydia trachomatis in a single reaction. J Clin Microbiol 44, 206-213.

Jalal, H., Stephen, H., Alexander, S., Carne, C. \& Sonnex, C. (2007a). Development of real-time PCR assays for genotyping of Chlamydia trachomatis. J Clin Microbiol 45, 2649-2653.

Jalal, H., Stephen, H., Bibby, D. F., Sonnex, C. \& Carne, C. A. (2007b). Molecular epidemiology of genital human papillomavirus and Chlamydia trachomatis among patients in Cambridge - will vaccines protect? Int J STD AIDS 18, 617-621.

Lysén, M., Osterlund, A., Rubin, C. J., Persson, T., Persson, I. \& Herrmann, B. (2004). Characterization of ompA genotypes by sequence analysis of DNA from all detected cases of Chlamydia trachomatis infections during 1 year of contact tracing in a Swedish County. J Clin Microbiol 42, 1641-1647.

Michel, C. E., Sonnex, C., Carne, C. A., White, J. A., Magbanua, J. P., Nadala, E. C., Jr \& Lee, H. H. (2007). Chlamydia trachomatis load at matched anatomic sites: implications for screening strategies. J Clin Microbiol 45, 1395-1402.

Millman, K., Black, C. M., Stamm, W. E., Jones, R. B., Hook, E. W., III, Martin, D. H., Bolan, G., Tavaré, S. \& Dean, D. (2006). Populationbased genetic epidemiologic analysis of Chlamydia trachomatis serotypes and lack of association between ompA polymorphisms and clinical phenotypes. Microbes Infect 8, 604-611.

Morré, S. A., Rozendaal, L., Van Valkengoed, I. G., Boeke, A. J., Van Voorst Vader, P. C., Schirm, J., de Blok, S., Van Den Hoek, J. A., Van Doornum, G. J. \& other authors (2000). Urogenital Chlamydia trachomatis serovars in men and women with a symptomatic or 
asymptomatic infection: an association with clinical manifestations? J Clin Microbiol 38, 2292-2296.

Oehme, A., Gaschler, G. \& Straube, E. (2003). Genotyping of Chlamydia trachomatis strains from cultured isolates and nucleic acid amplification test-positive specimens. Int J Med Microbiol 293, 225228.

Sayada, C., Denamur, E., Xerri, B., Orfila, J., Catalan, F. \& Elion, J. (1992). [Epidemiology of Chlamydia trachomatis using analysis of gene encoding of the major outer membrane protein]. Pathol Biol (Paris) 40, 583-589 (in French).

Suchland, R. J., Eckert, L. O., Hawes, S. E. \& Stamm, W. E. (2003). Longitudinal assessment of infecting serovars of Chlamydia trachomatis in Seattle public health clinics: 1988-1996. Sex Transm Dis 30, 357-361.

Van de Laar, M. J., Van Duynhoven, Y. T., Fennema, J. S., Ossewaarde, J. M., Van den Brule, A. J., Van Doornum, G. J., Coutinho, R. A. \& Van den Hoek, J. A. (1996). Differences in clinical manifestations of genital chlamydial infections related to serovars. Genitourin Med 72, 261-265.

Van Duynhoven, Y. T. H. P., Ossewaarde, J. M., Derksen-Nawrocki, R. P., Van der Meijden, W. I. \& Van de Laar, M. J. W. (1998). Chlamydia trachomatis genotypes: correlation with clinical manifestations of infection and patients' characteristics. Clin Infect Dis 26, 314322.

Wiggins, R., Graf, S., Low, N. \& Horner, P. J. for the Chlamydia Screening Studies (ClaSS) Study Group (2009). Real-time quantitative PCR to determine chlamydial load in men and women in a community setting. J Clin Microbiol 47, 1824-1829.

Workowski, K. A., Stevens, C. E., Suchland, R. J., Holmes, K. K., Eschenbach, D. A., Pettinger, M. B. \& Stamm, W. E. (1994). Clinical manifestations of genital infection due to Chlamydia trachomatis in women: differences related to serovar. Clin Infect Dis 19, 756760. 